\title{
Controlling topological polymorphism and dynamic behavior in ternary cocrystals
}

Filip Topić, ${ }^{1}$ Kari Rissanen, ${ }^{2}$ Tomislav Friščić ${ }^{1}$

${ }^{1}$ Department of Chemistry, McGill University, 801 Sherbrooke Street W., Montreal, H3A OB8, Canada. Email: filip.topic@mcgill.ca, tomislav.friscic@mcgill.ca

${ }^{2}$ Department of Chemistry, University of Jyväskylä, P.O. Box 35, FI-40014 Jyväskylä, Finland.

In the last two decades, co-crystals have emerged as a prominent topic in Crystal Engineering.[1] Whereas two-component (binary) co-crystals can often be prepared systematically, through rational designs that allow the fine tuning of their structure and properties, the synthesis of co-crystals consisting of three (ternary) or more components still remains a major challenge.

Recently, we reported a robust strategy for the preparation of ternary co-crystals using hydrogen and halogen bonds, combining thioureas, crown ethers and perfluorinated halogen bond donors.[2] These components form reliable and mutually orthogonal hydrogen (thioureas and crown ethers) and halogen bonds (thioureas with perfluorinated halogen bond donors).

In some cases, structures of the obtained cocrystals could be described as three-dimensional networks with thiourea molecules as nodes, and crown ethers and halogen bond donors as linkers. In particular, the 1:1:1 cocrystal of 18-crown-6 (18c6), $N$-methylthiourea (mtu) and octafluoro-1,4-diiodobenzene (ofib), (18c6)(mtu)(ofib), was found to appear in two polymorphic forms, differing only in their topology. On the other hand, the analogous cocrystal with thiourea (tu), (18c6)(tu)(ofib), only exhibits one topology. We successfully employed mechanochemistry not only to selectively prepare both polymorphs of $(\mathbf{1 8 c 6 )}(\mathrm{mtu})$ (ofib), but also to explore their dynamic behavior in the solid state. First, we were able to show that the two polymorphs are formed sequentially during a grinding experiment. Next, we found that $(\mathbf{1 8 c 6 )}(\mathrm{mtu})$ (ofib), upon grinding with tu, converts to $(\mathbf{1 8 c 6 ) ( t u ) ( o f i b ) . ~ O n ~ t h e ~ o t h e r ~ h a n d , ~ g r i n d i n g ~}$

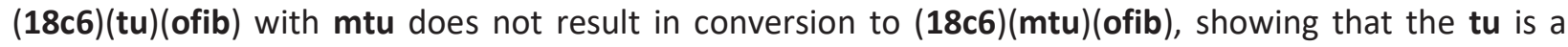
stronger linker than $\mathbf{m t u}$.

[1] C. B. Aakeröy, Acta Cryst., 2015, B71, 387-391.

[2] F. Topić and K. Rissanen, J. Am. Chem. Soc., 2016, 138, 6610-6616. 